ANNALES

POLONICI MATHEMATICI

$95.1(2009)$

\title{
Topological structure of solution sets to differential problems in Fréchet spaces
}

\author{
by A. BAzKOwska and G. GaBOR (Toruń)
}

\begin{abstract}
Using projective limit realizations of Fréchet spaces, we study the topological structure of solution sets for set differential equations and differential inclusions in Fréchet spaces. We apply suitable fixed point results for limit maps induced by maps of inverse systems.
\end{abstract}

1. Introduction. The theory of differential equations and inclusions has recently been developed in the direction of problems on nonnormed spaces. An important motivation is that several problems of modern theoretical physics, differential geometry and analysis require such a general setting. Fréchet spaces, i.e., metrizable locally convex complete topological vector spaces, are of special interest. There are essential differences between topologies of Banach and Fréchet spaces. For instance, each bounded subset of a Fréchet nonnormed space has empty interior. This causes several difficulties in studying properties of single-valued and set-valued maps in Fréchet spaces. Fortunately, since every Fréchet space can be viewed as a projective limit $\mathbb{F}=\lim _{\longleftarrow} \mathbb{E}^{i}$ of Banach spaces, the idea appeared to study a special type of maps, the so-called limit maps, and obtain results for them from the corresponding properties of maps between Banach spaces.

This technique has been used in fixed point theory (see, e.g., [16] and references therein) and in the theory of differential equations and inclusions ([13], [12]). The last two papers concern set differential equations

$$
\left(C_{F}\right)\left\{\begin{array}{l}
D_{H} x(t)=F(t, x(t)), \\
x(0)=x_{0} \in K(\mathbb{F}),
\end{array}\right.
$$

where $D_{H} x(t)$ is the Hukuhara differential on the space $K(\mathbb{F})$ of compact convex subsets of a Fréchet space $\mathbb{F}$. The authors examine a topology of $K(\mathbb{F})$ and prove the existence and uniqueness result for problem $\left(C_{F}\right)$. Analogous

2000 Mathematics Subject Classification: Primary 34A60; Secondary 46A13, 34G25.

Key words and phrases: set differential equations, Fréchet spaces, inverse systems, limit maps, topological structure. 
results in a Banach space are presented, e.g., in [18]. Note that the Hukuhara differentiability is one of several possibilities in differentiation of multivalued maps. We refer, e.g., to [5] or to [3] where slightly more general conical differentials were studied.

One of the aims of our paper is to develop the theory of set differential equations in Fréchet spaces and study the topological structure of the solution set to problem $\left(C_{F}\right)$ (Aronszajn type results). The general fixed point theorems for limit maps obtained in [1] and [10], and used in the proofs in our paper, enable one to get the results of [12] as easy consequences. Note that for every Banach factor $\mathbb{E}^{i}$ the metric space $K\left(\mathbb{E}^{i}\right)$ is a Banach semispace. This notion has recently been introduced by the first author in [2]. The background for our main result on solution sets for set differential equations in Fréchet spaces (Theorem 3.10) is given in this general Banach semispace setting.

The last part of the paper is devoted to evolution differential inclusions in Fréchet spaces. We also study the topological structure of solution sets by applying for every Banach factor the method due to Bressan, Cellina and Fryszkowski [4] adapted to this context by De Blasi, Pianigiani and Staicu [7]. The problem in Fréchet spaces meets essential obstacles and is not completely solved yet. We give two natural open problems at the end of Section 4, describing difficulties which are met when passing to the Fréchet case.

2. Fréchet spaces and fixed points of limit maps. Throughout this paper we use the following notation. $X$ is a complete metric space with a metric $d$, and $\mathbb{E}$ a real Banach space with a norm \|\| . By $2^{X}$ we denote the set of all nonempty subsets of $X$.

The space

$$
B(X)=\left\{A \in 2^{X}: A \text { is bounded and closed }\right\},
$$

endowed with the Pompeiu-Hausdorff metric

$$
D(A, B)=\max \{e(A, B), e(B, A)\}, \quad A, B \in B(X),
$$

where $e(A, B)=\sup _{x \in A} \inf _{y \in B} d(x, y)$, is a complete metric space.

Recall also the following notation:

$$
\begin{aligned}
& C(\mathbb{E})=\{A \in B(\mathbb{E}): A \text { is convex }\}, \\
& K(\mathbb{E})=\left\{A \in 2^{\mathbb{E}}: A \text { is compact, convex }\right\} .
\end{aligned}
$$

The space $K(\mathbb{E})$ endowed with the Pompeiu-Hausdorff metric is a complete metric space.

By an inverse system of topological spaces we mean a family

$$
S=\left\{X_{\alpha}, \pi_{\alpha}^{\beta}, \Sigma\right\},
$$

where $\Sigma$ is a set directed by a relation $\leq, X_{\alpha}$ is a topological (Hausdorff) 
space for every $\alpha \in \Sigma$, and $\pi_{\alpha}^{\beta}: X_{\beta} \rightarrow X_{\alpha}$ is a continuous mapping for any two $\alpha, \beta \in \Sigma$ such that $\alpha \leq \beta$. Moreover, $\pi_{\alpha}^{\alpha}=\operatorname{id}_{X}$ and $\pi_{\alpha}^{\beta} \circ \pi_{\beta}^{\gamma}=\pi_{\alpha}^{\gamma}$ for any $\alpha \leq \beta \leq \gamma$.

The limit of the inverse system $S$ (or the projective limit) is the subspace of the product $\prod_{\alpha \in \Sigma} X_{\alpha}$ defined by

$$
\lim _{\longleftarrow} S=\left\{\left(x_{\alpha}\right) \in \prod_{\alpha \in \Sigma} X_{\alpha}: \pi_{\alpha}^{\beta}\left(x_{\beta}\right)=x_{\alpha} \text { for all } \alpha \leq \beta\right\} .
$$

An element of $\lim S$ is called a thread or a fibre of the system $S$. If $\pi_{\alpha}$ : $\lim _{\longleftarrow} S \rightarrow X_{\alpha}$ is the restriction of the canonical projection $p_{\alpha}: \prod_{\alpha \in \Sigma} X_{\alpha} \rightarrow$ $\overleftarrow{X_{\alpha}}$, then $\pi_{\alpha}=\pi_{\alpha}^{\beta} \pi_{\beta}$ for any $\alpha \leq \beta$.

Now we recall some useful properties of limits of inverse systems.

Proposition 2.1 ([9]). Let $S=\left\{X_{\alpha}, \pi_{\alpha}^{\beta}, \Sigma\right\}$ be an inverse system.

1. The limit $\lim _{\longleftarrow} S$ is a closed subset of $\prod_{\alpha \in \Sigma} X_{\alpha}$.

2. If , for every $\alpha \in \Sigma, X_{\alpha}$ is

(i) compact, then $\lim _{\longleftarrow} S$ is compact;

(ii) compact and nonempty, then $\lim S$ is compact and nonempty;

(iii) a continuum, then $\lim S$ is a continuum;

(iv) compact and acyclic, then $\lim S$ is compact and acyclic;

(v) metrizable, $\Sigma$ is countable, and $\underset{\lim }{\longleftarrow} S$ is nonempty, then $\lim S$ is metrizable.

We also indicate a useful property for the case where $\Sigma=\mathbb{N}$.

Proposition 2.2 ([10, Proposition 3.2]). (Let $S=\left\{X_{n}, \pi_{n}^{p}, \mathbb{N}\right\}$ be an inverse system. If each $X_{n}$ is a compact $R_{\delta}$-set $\left({ }^{1}\right)$, then $\lim _{\longleftarrow} S$ is also $R_{\delta}$.

The following result is crucial to proving the main result in Section 3. We formulate it only for single-valued mappings.

Proposition 2.3 ([10, Theorem 6.9]). Let $S=\left\{X_{n}, \pi_{n}^{p}, \mathbb{N}\right\}$ be an inverse system, and $\phi: \lim S \rightarrow \lim S$ be a limit map induced by a map $\left\{\mathrm{id}, \phi_{n}\right\}$ of inverse systems, where $\phi_{n}: X_{n} \rightarrow X_{n}$. If the fixed point set of each $\phi_{n}$ is a compact $R_{\delta}$, then the fixed point set of $\phi$ is also $R_{\delta}$.

Let $\mathbb{F}$ be a Fréchet space with the topology defined by a sequence $\left\{p_{i}\right\}_{i \in \mathbb{N}}$ of seminorms. We may assume that $\left\{p_{i}\right\}_{i \in \mathbb{N}}$ is increasing, since in the other case we may define a new sequence $\left\{q_{i}\right\}_{i \in \mathbb{N}}$ by putting

$$
q_{i}=p_{1}+\cdots+p_{i}
$$

which defines the same topology on $\mathbb{F}$.

$\left({ }^{1}\right)$ We say that a metric space is an $R_{\delta}$-set if it is homeomorphic to the intersection of a countable decreasing sequence of absolute retracts. 
Denote the completion of the quotient $\mathbb{F} / \operatorname{Ker} p_{i}$ by $\mathbb{E}^{i}$, and by $\varrho^{j i}$ the connecting morphism

$$
\varrho^{j i}: \mathbb{E}^{j} \rightarrow \mathbb{E}^{i}, \quad\left[x+\operatorname{Ker} p_{j}\right]_{j} \mapsto\left[x+\operatorname{Ker} p_{i}\right]_{i}, \quad j \geq i,
$$

where $[\cdot]_{i}$ stands for the corresponding equivalence class; it is easy to show that $\varrho^{j i}$ is a continuous linear mapping. Then $\mathbb{F}$ coincides, up to isomorphism, with the limit of the inverse system

$$
\left\{\mathbb{E}^{i}, \varrho^{j i}\right\} .
$$

Note that the canonical projections

$$
\varrho^{i}: \mathbb{F} \equiv \lim _{\longleftarrow} \mathbb{E}^{i} \rightarrow \mathbb{E}^{i}, \quad x \mapsto\left[x+\operatorname{Ker} p_{i}\right]_{i}, \quad i \in \mathbb{N},
$$

become isometries in the sense that

$$
p_{i}(x)=\left\|\left[x+\operatorname{Ker} p_{i}\right]_{i}\right\|_{\mathbb{E}^{i}}=\left\|\varrho^{i}(x)\right\|_{\mathbb{E}^{i}},
$$

where $\|\cdot\|_{\mathbb{E}^{i}}$ stands for the norm in $\mathbb{E}^{i}$. We also recall that the mappings $\varrho^{j i}$ and $\varrho^{i}$ satisfy the conditions

$$
\varrho^{j i} \circ \varrho^{j}=\varrho^{i}, \quad \varrho^{i k} \circ \varrho^{j i}=\varrho^{j k},
$$

for all $i, j, k \in \mathbb{N}$ with $j \geq i \geq k$.

Now we list some useful properties of $K(\mathbb{F})$.

Proposition 2.4 ([13, Proposition 3.3]). $K(\mathbb{F})$ is closed under the operations of addition and scalar multiplication.

Let $\left\{\mathbb{E}^{i}, \varrho^{j i}\right\}_{i, j \in \mathbb{N}}$ be an inverse system of Banach spaces that realizes the Fréchet model space $\mathbb{F}$. Then the following result also holds.

Proposition 2.5 ([13, Proposition 3.1]). The family $\left\{K\left(\mathbb{E}^{i}\right)\right\}_{i \in \mathbb{N}}$ forms an inverse system of metric spaces whose limit coincides (set-theoretically) with $K(\mathbb{F})$ :

$$
K(\mathbb{F})=\lim _{\longleftarrow} K\left(\mathbb{E}^{i}\right) .
$$

Note that this inverse system is defined by

$$
\psi^{j i}: K\left(\mathbb{E}^{j}\right) \rightarrow K\left(\mathbb{E}^{i}\right), \quad A \mapsto \varrho^{j i}(A), \quad j \geq i .
$$

Denote by $\psi^{i}: K(\mathbb{F}) \rightarrow K\left(\mathbb{E}^{i}\right)$ the corresponding projections.

The above described structure of $K(\mathbb{F})$ makes it possible to adapt the notion of Hausdorff distance to the projective limit approach.

Definition 2.6 ([13]). For every $A, B \in K(\mathbb{F})$ we define:

(i) the $i$-distance of $x$ to $A$ :

$$
d^{i}(x, A)=\inf \left\{p_{i}(x-a): a \in A\right\} ;
$$

(ii) the $i$-separation of $A, B \in K(\mathbb{F})$ :

$$
d_{H}^{i}(B, A)=\sup \left\{d^{i}(b, A): b \in B\right\} ;
$$


(iii) the $i$-Hausdorff distance or $i$-semidistance of $A$ and $B$ :

$$
D^{i}(B, A)=\max \left\{d_{H}^{i}(B, A), d_{H}^{i}(A, B)\right\} .
$$

It is worth pointing out that if $A=\lim A^{i}$ and $B=\lim ^{i} B^{i}$ for $A^{i}, B^{i} \in$ $K\left(\mathbb{E}^{i}\right)$, then $D^{i}(A, B)=D^{\mathbb{E}^{i}}\left(A^{i}, B^{i}\right)$, where $D^{\mathbb{E}^{i}}$ stands for the Hausdorff metric in $\mathbb{E}^{i}$ for $i \in \mathbb{N}$. Since the space $K(\mathbb{F})$ is the projective limit of the metrizable spaces $K\left(\mathbb{E}^{i}\right)$, it is also metrizable with the standard projective limit metric, or equivalently, by the family of semimetrics $D^{i}$. This topology is complete and separable.

Proposition 2.7. The family $\left\{C\left([a, b], K\left(\mathbb{E}^{i}\right)\right)\right\}_{i \in \mathbb{N}}$ forms an inverse system of metric spaces whose limit coincides with $C([a, b], K(\mathbb{F}))$,

$$
C([a, b], K(\mathbb{F}))=\lim _{\longleftarrow} C\left([a, b], K\left(\mathbb{E}^{i}\right)\right) .
$$

Proof. We define mappings

$$
\Psi^{j i}: C\left([a, b], K\left(\mathbb{E}^{j}\right)\right) \rightarrow C\left([a, b], K\left(\mathbb{E}^{i}\right)\right)
$$

for $j \geq i$ as follows. For $f \in C\left([a, b], K\left(\mathbb{E}^{j}\right)\right)$ let $\Psi^{j i}(f)(t)=\psi^{j i}(f(t))$ for every $t \in[a, b]$, where $\psi^{j i}$ is as above.

Since the $\psi^{j i}$ are connecting morphisms of an inverse system, the maps $\Psi^{j i}$ also satisfy

$$
\Psi^{i k} \circ \Psi^{j i}=\Psi^{j k}, \quad j \geq i \geq k .
$$

Moreover, each $\Psi^{j i}$ is continuous with respect to the topologies induced by the Chebyshev metric on $\left(C\left([a, b], K\left(\mathbb{E}^{i}\right)\right)\right.$ defined by

$$
D_{C}^{\mathbb{E}^{i}}(f, g)=\sup _{t \in[a, b]} D^{\mathbb{E}^{i}}(f(t), g(t)) .
$$

Indeed,

$$
D_{C}^{\mathbb{E}^{i}}\left(\Psi^{j i}(f), \Psi^{j i}(g)\right)=\sup _{t \in[a, b]} D^{\mathbb{E}^{i}}\left(\psi^{j i}(f(t)), \psi^{j i}(g(t))\right) .
$$

Since, as we can easily check, $D^{\mathbb{E}^{i}}\left(\psi^{j i}(A), \psi^{j i}(B)\right) \leq D^{\mathbb{E}^{j}}(A, B)$ for any $A, B \in K\left(\mathbb{E}^{i}\right)$, we have

$$
\sup _{t \in[a, b]} D^{\mathbb{E}^{i}}\left(\psi^{j i}(f(t)), \psi^{j i}(g(t))\right) \leq \sup _{t \in[a, b]} D^{\mathbb{E}^{j}}(f(t), g(t))=D_{C}^{\mathbb{E}^{j}}(f, g) .
$$

Consequently, $\Psi^{j i}$ is (uniformly) continuous. Hence, $\left\{C\left([a, b], K\left(\mathbb{E}^{i}\right)\right), \Psi^{j i}\right\}$ is an inverse system. We define $\theta: C([a, b], K(\mathbb{F})) \rightarrow \lim C\left([a, b], K\left(\mathbb{E}^{i}\right)\right)$ by $\theta(f)(t):=\left(\psi^{i}(f(t))\right)$. It can be seen that $\theta$ is injective. Moreover, for every fibre $f=\left(f^{i}\right) \in \lim _{t} C\left([a, b], K\left(\mathbb{E}^{i}\right)\right)$, since each $f^{i}$ is continuous and $f^{i}(t)$ is a fibre for every $t \in[a, b], f$ can be viewed as a continuous map from $[a, b]$ to $\lim _{L} K\left(\mathbb{E}^{i}\right)=K(\mathbb{F})$. Thus $\theta$ is also surjective. Finally, it is an isometry, since we consider the projective metrics in $K(\mathbb{F})$ and $\lim _{\longleftarrow} C\left([a, b], K\left(\mathbb{E}^{i}\right)\right)$. 
3. Solution sets for set differential equations in Banach and Fréchet spaces. Let $M$ be a nonempty set equipped with two operations:

$$
+: M \times M \rightarrow M \text { and } \circ:[0, \infty) \times M \rightarrow M .
$$

We use the following notations: $+(x, y)=x+y, \circ(\lambda, x)=\lambda \circ x$.

Definition 3.1. A triple $(M,+, \circ)$ is a semilinear space if the following conditions are satisfied for any $a, b, c \in M$ and $\lambda, \mu \geq 0$ :

(i) $a+b=b+a$,

(ii) $(a+b)+c=a+(b+c)$,

(iii) $\exists_{0 \in M} a+0=a$,

(iv) $1 \circ a=a$,

(v) $(\lambda \cdot \mu) \circ a=\lambda \circ(\mu \circ a)$,

(vi) $(\lambda+\mu) \circ a=\lambda \circ a+\mu \circ a$,

(vii) $\lambda \circ(a+b)=\lambda \circ a+\lambda \circ b$.

For simplicity of notation we write $\lambda a$ instead of $\lambda \circ a$.

Definition 3.2. Let $(M,+, \circ)$ be a semilinear space with a complete metric $d$ such that the operations of addition and multiplication by a nonnegative scalar are continuous. We say that $(M,+, \circ, d, i)$ is a Banach semispace if there exists a real Banach space $\mathbb{E}$ and a function $i: M \rightarrow \mathbb{E}$ such that

$$
i(\lambda a+\mu b)=\lambda i(a)+\mu i(b), \quad\|i(a)-i(b)\|=d(a, b)
$$

for all $a, b \in M$ and $\lambda, \mu \geq 0$.

Obviously, every Banach space and every closed convex cone in a Banach space space are Banach semispaces. Below we give several other examples.

EXAMPLE 3.3. It is known [17] that the space $K(\mathbb{E})$ equipped with the natural algebraic operations and multiplication by nonnegative scalars becomes a semilinear space which can be embedded as a complete cone into a Banach space. This means that we have $i: K(\mathbb{E}) \rightarrow \widetilde{\mathbb{E}}$, where $\widetilde{\mathbb{E}}$ is a real Banach space that satisfies the following conditions:

(i) $i$ is an isometry,

(ii) $i(\lambda A+\mu B)=\lambda i(A)+\mu i(B)$ for $A, B \in K(\mathbb{E})$ and $\lambda, \mu \in[0, \infty)$. Consequently, $K(\mathbb{E})$ is a Banach semispace.

EXAMPLE 3.4 ([2]). Using the Rådström-Hörmander [14] embedding we shall show that the space $C(\mathbb{E})$ with addition and multiplication by nonnegative scalars defined by

$$
X+Y=\overline{\{x+y: x \in X, y \in Y\}} \quad \text { and } \quad \lambda X=\{\lambda x: x \in X\}
$$

is a Banach semispace. 
Let $\mathbb{E}^{*}$ be the topological dual of $\mathbb{E}$. Following Hörmander (see [13]) we define

$\mathbf{H}=\left\{q: \mathbb{E}^{*} \rightarrow \mathbb{R}: q\right.$ is positively homogeneous and norm-continuous $\}$. Then $\mathbf{H}$ equipped with the norm

$$
\|q\|_{\mathbf{H}}=\sup _{\left\|x^{*}\right\| \leq 1}\left\|q\left(x^{*}\right)\right\|, \quad q \in \mathbf{H},
$$

is a real Banach space.

For $X \in C(\mathbb{E})$, let $q_{X}: \mathbb{E}^{*} \rightarrow \mathbb{R}$ be the support function of $X$, given by

$$
q_{X}\left(x^{*}\right)=\sup _{x \in X} x^{*}(x), \quad x^{*} \in \mathbb{E}^{*} .
$$

Then the function $j: C(\mathbb{E}) \rightarrow \mathbf{H}$ defined by

$$
j(X)=q_{X}, \quad X \in C(\mathbb{E}),
$$

establishes an isomorphism between $C(\mathbb{E})$ and the positive convex cone

$$
V=\left\{q_{X} \in \mathbf{H}: X \in C(\mathbb{E})\right\} .
$$

Moreover, $j$ is an isometry, which means

$$
\|j(X)-j(Y)\|_{\mathbf{H}}=H(X, Y), \quad X, Y \in C(\mathbb{E}) .
$$

EXAmple $3.5([2])$. Let $A \subset \mathbb{E}$. We say that $A$ is starshaped with respect to 0 if for every $x \in A$ we have $\lambda x \in A$ for all $\lambda \in[0,1]$. Consider the family of sets

$$
C_{0-\operatorname{sh}}(\mathbb{E})
$$

$=\left\{X \in 2^{\mathbb{E}}: X\right.$ is closed, bounded and starshaped with respect to 0$\}$. We define addition and multiplication by nonnegative scalars by $X+Y=$

$$
\begin{aligned}
& \overline{\left\{x+y: x \in X, y \in Y \text { with } x=\lambda s, y=\mu s \text { for some } \lambda, \mu \geq 0 \text { and } s \in S^{\mathbb{E}}\right\}} \text {, } \\
& \lambda X=\{\lambda x: x \in X\}
\end{aligned}
$$

for $X, Y \in C_{0 \text {-sh }}(\mathbb{E})$ and $\lambda \geq 0$. Here $S^{\mathbb{E}}$ denotes the unit sphere in $\mathbb{E}$. The space $C_{0 \text {-sh }}(\mathbb{E})$ is endowed with the metric

$$
H_{0-\mathrm{sh}}(X, Y)=\max \left\{\sup _{x \in X} d_{0-\mathrm{sh}}(x, Y), \sup _{y \in Y} d_{0-\operatorname{sh}}(y, X)\right\}
$$

where

$$
d_{0-\mathrm{sh}}(x, Y)=\inf \{\|x-y\|: y \in Y, y=\lambda x \text { for some } \lambda \geq 0\} .
$$

It is easy to see that, just as $(C(\mathbb{E}), H),\left(C_{0 \text {-sh }}(\mathbb{E}), H_{0 \text {-sh }}(\mathbb{E})\right)$ is a complete metric space.

Recall that the space

$$
B\left(S^{\mathbb{E}}, R\right)=\left\{f: S^{\mathbb{E}} \rightarrow \mathbb{R}: f \text { is bounded }\right\}
$$


with the norm $\|f\|=\sup _{s \in S^{\mathbb{E}}}|f(s)|$ is a real Banach space. Now we can define

$$
i: C_{0 \text {-sh }}(\mathbb{E}) \rightarrow B\left(S^{\mathbb{E}}, \mathbb{R}\right)
$$

by putting

$$
i(X)=f_{X}
$$

where $f_{X}(s)=\sup \{\lambda: \lambda s \in X\}$ for $X \in C_{0 \text {-sh }}(\mathbb{E})$. It is easy to show that

$$
i(\lambda X+\mu Y)=\lambda i(X)+\mu i(Y), \quad\|i(X)-i(Y)\|=H_{0-\text { sh }}(X, Y),
$$

for all $X, Y \in C_{0 \text {-sh }}(\mathbb{E})$ and $\lambda, \mu \geq 0$.

Thus we have shown that $C_{0 \text {-sh }}(\mathbb{E})$ is a Banach semispace.

EXAmple $3.6([2])$. Let $(M,+, \circ, d, i)$ be a Banach semispace associated with a real Banach space $\mathbb{E}$ and a homeomorphism $i: M \rightarrow \mathbb{E}$ as in Definition 3.2. Let $K$ be a nonempty metric space. Given two functions $x, y: K \rightarrow M$ and $\lambda \geq 0$, the sum $x+y: K \rightarrow M$ and the product $\lambda x: K \rightarrow M$ are defined by

$$
(x+y)(t)=x(t)+y(t), \quad \lambda x(t)=\lambda \circ x(t), \quad t \in K .
$$

Now put

$$
B(K, M)=\{x: K \rightarrow M: x \text { is continuous and bounded }\}
$$

end equip $B(K, M)$ with the metric

$$
d_{B}(x, y)=\sup _{t \in M} d(x(t), y(t)), \quad x, y \in B(K, M) .
$$

As $(M, d)$ is complete, for $x, y \in B(K, M)$ and $\lambda \geq 0$ we have $x+y \in$ $B(K, M)$ and $\lambda x \in B(K, M)$. Moreover, if $x, y \in B(K, M)$ and $\lambda, \mu \geq 0$, then all the conditions of Definition 3.1 are satisfied, where 0 stands for the map identically zero on $K$.

Next put

$$
C(M, \mathbb{E})=\{\xi: M \rightarrow \mathbb{E}: \xi \text { is continuous and bounded }\},
$$

equipped with the norm

$$
\|\xi\|_{C}=\sup _{t \in M}\|\xi(t)\|_{\mathbb{E}} .
$$

Clearly, $\left(C(M, \mathbb{E}),\|\|_{C}\right)$ is a real Banach space.

Now denote by $I: B(K, M) \rightarrow C(M, \mathbb{E})$ the map which associates to each $x \in B(K, M)$ the element $I(x)$ of $C(M, \mathbb{E})$ defined by

$$
(I(x))(t)=i(x(t)), \quad t \in M .
$$

Observe that $I(x) \in C(M, \mathbb{E})$. The set

$$
V=\{\xi \in C(M, \mathbb{E}): \text { there exists } \xi \in B(K, M) \text { such that } I(x)=\xi\}
$$


is a convex cone contained in $C(M, \mathbb{E})$. More precisely, $I: B(K, M) \rightarrow$ $C(M, \mathbb{E})$ is an isometric map onto the positive convex cone $V$ in $C(M, \mathbb{E})$, namely, for all $x, y \in B(K, M)$ and $\lambda, \mu \geq 0$ we have

$$
I(\lambda x+\mu y)=\lambda I(x)+\mu I(y), \quad\|I(x)-I(y)\|_{C}=d_{B}(x, y) .
$$

Consequently, $B(K, M)$ is a Banach semispace.

Definition 3.7. We say that a map $x:(a, b) \rightarrow M$ has the Hukuhara derivative at a point $t_{0} \in(a, b)$ if

$$
D_{\mathrm{H}} x\left(t_{0}^{+}\right):=\lim _{h \rightarrow 0^{+}} \frac{x\left(t_{0}+h\right)-x\left(t_{0}\right)}{h}, \quad D_{\mathrm{H}} x\left(t_{0}^{-}\right):=\lim _{h \rightarrow 0^{+}} \frac{x\left(t_{0}\right)-x\left(t_{0}-h\right)}{h}
$$

exist in the topology of $M$ and are equal. We denote the Hukuhara derivative by $D_{\mathrm{H}} x\left(t_{0}\right)$.

The existence of, e.g., $x\left(t_{0}+h\right)-x\left(t_{0}\right)$ means that there exists an element $m \in M$ such that $m+x\left(t_{0}\right)=x\left(t_{0}+h\right)$. For $M=K(\mathbb{E})$ this element is known as the Hukuhara difference (or geometrical difference, see [18]).

For $x:[a, b] \rightarrow M$, if $D_{\mathrm{H}} x(t)$ exists for any $t \in(a, b)$, and $D_{\mathrm{H}} x\left(a^{+}\right)$and $D_{\mathrm{H}} x\left(b^{-}\right)$exist, we say that $x$ is Hukuhara differentiable on $[a, b]$.

An important example of a Hukuhara differentiable map is given in

Proposition 3.8. Let $F:[a, b] \rightarrow M$ be an integrable map (in the sense of Bochner). Then $G:[a, b] \rightarrow M$ given by

$$
G(t)=\int_{a}^{t} F(s) d s
$$

is Hukuhara differentiable, and $D_{\mathrm{H}} G(t)=F(t)$ a.e. on $[a, b]$.

A map $F:[0, a] \times M \rightarrow M$ is called a Carathéodory map if:

(i) $t \mapsto F(t, x)$ is strongly measurable for every $x \in M$,

(ii) $x \mapsto F(t, x)$ is continuous for $t \in[0, a]$,

(iii) $d(0, F(t, x)) \leq \mu(t)$ for every $t \in[0, a], x \in M$, where $\mu:[0, a] \rightarrow$ $[0, \infty)$ is an integrable function.

Now consider the Cauchy problem

$$
\left(C_{F}\right)\left\{\begin{array}{l}
D_{\mathrm{H}} x(t)=F(t, x(t)), \\
x(0)=x_{0}
\end{array}\right.
$$

where $F:[0, a] \times M \rightarrow M$ is a Carathéodory function and $x_{0} \in M$.

By a solution of the Cauchy problem $\left(C_{F}\right)$ we mean an absolutely continuous function $x:[0, a] \rightarrow M$ with $x(0)=x_{0}$ satisfying $D_{\mathrm{H}} x(t)=F(t, x(t))$ for a.e. $t \in[0, a]$.

Before we present the main theorem we need some topological notions. 
Let $A$ be a bounded subset of a Banach space $\mathbb{E}$. Then $\alpha(A)=\inf \{r>0: A$ can be covered by finitely many sets of diameter $\leq r\}$

is called the (Kuratowski) measure of noncompactness. Some of its properties are listed in the following proposition.

Proposition 3.9 ([8]). Let $A, B$ be bounded subsets of a Banach space $\mathbb{E}$. Then

(1) $\alpha(A)=0$ iff $\bar{A}$ is compact,

(2) $\alpha(t \cdot A)=|t| \alpha(A)$ for $t \in R$,

(3) $\alpha(A+B) \leq \alpha(A)+\alpha(B)$,

(4) $\alpha(\overline{\operatorname{conv} A})=\alpha(A)$,

(5) if $A \subset B$, then $\alpha(A) \leq \alpha(B)$,

(6) if $D \subset C([a, b], \mathbb{E})$ is a bounded set of equicontinuous functions, then $\alpha(D)=\sup \{\alpha(\{x(t): x \in D\}): t \in[a, b]\}$,

A function $\omega:[0, a] \times \mathbb{R}_{+} \rightarrow \mathbb{R}_{+}$is called a Kamke function if:

(i) $t \mapsto \omega(t, x)$ is measurable for every $x \in \mathbb{R}$,

(ii) $x \mapsto \omega(t, x)$ is continuous for every $t \in[0, a]$,

(iii) the inequality $u(t) \leq \int_{0}^{t} \omega(s, u(s)) d s$ for every $t \in[0, a]$ has only the trivial solution, that is, $u(t)=0$ for every $t \in[0, a]$.

Now we can formulate a theorem on the topological structure of the solution set to $\left(C_{F}\right)$ in Banach semispaces:

Theorem $3.10([2])$. Let $M$ be a Banach semispace, and $F:[0, a] \times M \rightarrow$ $M$ be a Carathéodory function with $\mu(t)=r>0$ and

$$
\lim _{\delta \rightarrow 0^{+}} \alpha\left(F\left(I_{t, \delta} \times A\right)\right) \leq \omega(t, \alpha(A))
$$

for every bounded $A \subset M$ and a.e. $t \in[0, a]$, where $I_{t, \delta}=[t-\delta, t+\delta] \cap[0, a]$. Then the set of solutions of $\left(C_{F}\right)$ is a nonempty $R_{\delta}$-set.

The proof is based on the following reformulation of the Browder-Gupta theorem:

Theorem 3.11 (cf. [6, Theorem 7]). Let $X$ be a metric space, $\mathbb{E}$ be a Banach space and $f: X \rightarrow \mathbb{E}$ be a continuous map such that:

- $f$ is proper at $0 \in \mathbb{E}$,

- for every $\varepsilon>0$ there exists a continuous proper map $f_{\varepsilon}: X \rightarrow Y$ satisfying:

(i) $\left\|f(x)-f_{\varepsilon}(x)\right\|<\varepsilon$ for every $x \in X$,

(ii) $f_{\varepsilon}$ restricts to a homeomorphism of $f_{\varepsilon}^{-1}(B(0, \varepsilon))$ onto $B(0, \varepsilon)$.

Then $f^{-1}(0)$ is an $R_{\delta}$-set. 
We also need a lemma:

Lemma $3.12([15])$. Let $\widetilde{F}:[0, a] \times i(M) \rightarrow \mathbb{E}$ satisfy the conditions of Theorem 3.10 and $\left\{x_{n}: n \in \mathbb{N}\right\} \subset C([0, a], i(M))$ be a bounded equicontinuous set. Then, for every $T \in[0, a]$,

$$
\alpha\left(\left\{\int_{0}^{T} \widetilde{F}\left(s, x_{n}(s)\right) d s: n \in \mathbb{N}\right\}\right) \leq \int_{0}^{T} \omega\left(s, \alpha\left(\left\{x_{n}(s): n \in \mathbb{N}\right\}\right)\right) d s .
$$

Sketch of proof of Theorem 3.10. We divide the proof into three parts.

(a) First observe that $\widetilde{F}:[0, a] \times i(M) \rightarrow \mathbb{E}, \widetilde{F}(t, y):=i\left(F\left(t, i^{-1}(y)\right)\right)$, also satisfies the assumptions of the theorem.

(b) We define the integral operator $\mathcal{G}: C([0, a], i(M)) \rightarrow C([0, a], \mathbb{E})$ by putting

$$
\mathcal{G}(x)(t)=i\left(x_{0}\right)+\int_{0}^{t} \widetilde{F}(s, x(s)) d s .
$$

It is sufficient to show that the set of fixed points of $\mathcal{G}$ is a nonempty $R_{\delta}$-set.

Let $f: C([0, a], i(M)) \rightarrow C([0, a], \mathbb{E})$ be defined by putting

$$
f(x)(t):=x(t)-\mathcal{G}(x)(t) .
$$

We have

$$
f^{-1}(\{0\})=\operatorname{Fix} \mathcal{G} .
$$

It is easily seen that $\mathcal{G}$ and $f$ are continuous. We show that $f$ satisfies the assumptions of Theorem 3.11. So we have to construct a sequence $\left\{f_{n}\right\}$ of continuous, proper and injective functions uniformly convergent to $f$. Let $\mathcal{G}_{n}: C([0, a], i(M)) \rightarrow C([0, a], \mathbb{E})$ be defined as follows:

$$
\mathcal{G}_{n}(x)(t):= \begin{cases}i\left(x_{0}\right) & t \leq 1 / n \\ \mathcal{G}(x)(t-1 / n) & t \geq 1 / n\end{cases}
$$

and set $f_{n}(x)(t):=x(t)-\mathcal{G}_{n}(x)(t)$. Obviously, $f_{n}$ is continuous and by easy calculations we find that it is injective. Now we have to check that $f_{k}$ is proper for every $k \in \mathbb{N}$. Pick a compact set $A \subset C([0, a], \mathbb{E})$ and any sequence $\left\{x_{n}\right\} \subset f_{k}^{-1}(A)$. Then $\left\{x_{n}: n \in \mathbb{N}\right\}$ is a bounded equicontinuous set. By Proposition 3.9 we have

$$
\alpha\left(\left\{x_{n}: n \in \mathbb{N}\right\}\right)=\sup _{t \in[0, a]} \alpha\left(\left\{x_{n}(t): n \in \mathbb{N}\right\}\right) .
$$

There is no loss of generality in assuming that $\left\{x_{n}(t)-\mathcal{G}_{k}\left(x_{n}\right)(t)\right\}$ is convergent. As a result we obtain

$$
\begin{aligned}
\alpha\left(\left\{x_{n}(t): n \in \mathbb{N}\right\}\right) & =\alpha\left(\left\{\mathcal{G}_{k}\left(x_{n}\right)(t)+x_{n}(t)-\mathcal{G}_{k}\left(x_{n}\right)(t): n \in \mathbb{N}\right\}\right) \\
\leq & \alpha\left(\left\{\mathcal{G}_{k}\left(x_{n}\right)(t): n \in \mathbb{N}\right\}\right)+\alpha\left(\left\{x_{n}(t)-\mathcal{G}_{k}\left(x_{n}\right)(t): n \in \mathbb{N}\right\}\right) \\
& =\alpha\left(\left\{\mathcal{G}_{k}\left(x_{n}\right)(t): n \in \mathbb{N}\right\}\right) .
\end{aligned}
$$


For $t \leq 1 / k$ we have

$$
\alpha\left(\left\{\mathcal{G}_{k}\left(x_{n}\right)(t): n \in \mathbb{N}\right\}\right)=\alpha\left(\left\{i\left(x_{0}\right)\right\}\right)=0 .
$$

If $t \geq 1 / k$, then

$$
\begin{aligned}
\alpha\left(\left\{\mathcal{G}_{k}\left(x_{n}\right)(t): n \in \mathbb{N}\right\}\right) & =\alpha\left(\left\{i\left(x_{0}\right)+\int_{0}^{t-1 / k} \widetilde{F}\left(s, x_{n}(s)\right) d s: n \in \mathbb{N}\right\}\right) \\
& =\alpha\left(\left\{\int_{0}^{t-1 / k} \widetilde{F}\left(s, x_{n}(s)\right) d s: n \in \mathbb{N}\right\}\right) .
\end{aligned}
$$

By Lemma 3.12 we have

$$
\begin{aligned}
\alpha\left(\left\{\int_{0}^{t-1 / k} \widetilde{F}\left(s, x_{n}(s)\right) d s: n \in \mathbb{N}\right\}\right) & \leq \int_{0}^{t-1 / k} \omega\left(s, \alpha\left(\left\{x_{n}(s): n \in \mathbb{N}\right\}\right)\right) d s \\
& \leq \int_{0}^{t} \omega\left(s, \alpha\left(\left\{x_{n}(s): n \in \mathbb{N}\right\}\right)\right) d s .
\end{aligned}
$$

Thus we obtain the inequality

$$
\alpha\left(\left\{x_{n}(t): n \in \mathbb{N}\right\}\right) \leq \int_{0}^{t} \omega\left(s, \alpha\left(\left\{x_{n}(s): n \in \mathbb{N}\right\}\right)\right) d s .
$$

This holds only for $\alpha\left(\left\{x_{n}(t): n \in \mathbb{N}\right\}\right)=0$, which is equivalent to $f_{k}$ being proper. By compactness and injectivity, $f_{n}$ is an open map.

We now show that $f_{k}$ is a closed map. Let $A$ be a closed subset of $C([0, a], i(M))$. To show that $f_{k}(A)$ is also closed, let $\left\{x_{n}(t)-\mathcal{G}_{k}\left(x_{n}(t)\right)\right\} \subset$ $f(A)$ be a convergent sequence. As above, we obtain (1), and so

$$
\alpha\left(\left\{x_{n}(t): n \in \mathbb{N}\right\}\right)=0 .
$$

Consequently, $x_{n}$ has a subsequence, denoted also by $\left\{x_{n}\right\}$, convergent to $x_{0} \in A$, and by the continuity of $f_{k}$, the sequence $f_{k}\left(x_{n}\right)$ converges to $f_{k}\left(x_{0}\right) \in f_{k}(A)$.

(c) Now we prove that $f^{-1}(0)$ is nonempty. We construct the successive approximations by putting

$$
x_{n}(t)= \begin{cases}i\left(x_{0}\right)+\widetilde{F}\left(0, i\left(x_{0}\right)\right) t, & 0 \leq t \leq a / n, \\ i\left(x_{0}\right)+\widetilde{F}\left(0, i\left(x_{0}\right)\right) \frac{a}{n}+\int_{0}^{t-a / n} \widetilde{F}\left(s, x_{n}(s)\right) d s, & a / n \leq t \leq a .\end{cases}
$$

We show that $\alpha\left(\left\{x_{n}(t): n \in \mathbb{N}\right\}\right)=0$. Obviously, by definition, $\left\{x_{n}: n \in \mathbb{N}\right\}$ is a bounded equicontinuous set. For any $\varepsilon>0$, select $k$ such that $2 r a / k \leq \varepsilon$. 
Then

$$
\begin{aligned}
\alpha\left(\left\{x_{n}(t): n \geq k\right\}\right) & \leq \alpha\left(\left\{\int_{0}^{t} \widetilde{F}\left(s, x_{n}(s)\right) d s: n \geq k\right\}\right)+\frac{2 r a}{k} \\
& \leq \alpha\left(\left\{\int_{0}^{t} \widetilde{F}\left(s, x_{n}(s)\right) d s: n \geq k\right\}\right)+\varepsilon .
\end{aligned}
$$

By Lemma 3.12, $\alpha\left(\left\{x_{n}(t): n \geq k\right\}\right) \leq \int_{0}^{t} \omega\left(s, \alpha\left(\left\{x_{n}(s): n \geq k\right\}\right) d s+\varepsilon\right.$. Consequently, we may assume that $\left\{x_{n}\right\}$ is convergent. We have to show that its limit $x:[0, a] \rightarrow i(K(M))$ corresponds to a solution of $\left(C_{F}\right)$.

For $t \in(0, a]$ we calculate

$$
\begin{aligned}
\| x(t)-i\left(x_{0}\right)- & \int_{0}^{t} \widetilde{F}(s, x(s)) d s \| \\
\leq & \left\|x(t)-x_{n}(t)\right\|+\int_{0}^{t-a / n}\left\|\widetilde{F}\left(s, x_{n}(s)\right)-\widetilde{F}(s, x(s))\right\| d s \\
& +\int_{t-a / n}^{t}\left\|\widetilde{F}(s, x(s))-\widetilde{F}\left(0, i\left(x_{0}\right)\right)\right\| d s .
\end{aligned}
$$

By the definition of $x$ and the Lebesgue theorem the right hand side of the above inequality converges to 0 .

Hence $f^{-1}(0) \neq \emptyset$, i.e., $\mathcal{G}$ has a fixed point. By definition, each $\mathcal{G}_{k}$ also has a fixed point, and so $f_{k}^{-1}(0) \neq \emptyset$. Summarizing, every $f_{k}$ is proper, injective, closed and $f_{k}^{-1}(0) \neq \emptyset$. Therefore the set $f_{k}(C([0, a], i(M)))$ is open and $0 \in f_{k}(C([0, a], i(M)))$. Pick $\varepsilon_{n}$ such that $B\left(0, \varepsilon_{n}\right) \subset f_{n}(C([0, a], i(M)))$. Of course, $f_{n}$ establishes a homeomorphism of $f_{n}^{-1}\left(B\left(0, \varepsilon_{n}\right)\right)$ onto $B\left(0, \varepsilon_{n}\right)$. By Theorem 3.11, $f^{-1}(0)$ is an $R_{\delta^{-} \text {set. }}$

Let $\mathbb{F}$ be an arbitrary Fréchet space represented as a projective limit of Banach spaces $\mathbb{F}=\lim \left\{\mathbb{E}^{i}, \varrho^{j i}\right\}$. Consider the Cauchy problem $\left(C_{F}\right)$ with $F \in C([0, a] \times K(\mathbb{F}), \overleftarrow{K}(\mathbb{F}))$ and $x_{0} \in K(\mathbb{F})$.

As in Definition 3.7, we can define the Hukuhara differential for $x$ : $[a, b] \rightarrow K(\mathbb{F})$, and since the maps $\varrho^{i j}$ are continuous and linear, we can prove (cf. [13, Proposition 4.5]) that $x=\lim _{\longleftarrow} x^{i}:[a, b] \rightarrow K(\mathbb{F})$ is Hukuhara differentiable at $t_{0} \in[a, b]$ (i.e. $D_{\mathrm{H}} x\left(t_{0}\right)$ exists) if and only if each $x^{i}:[a, b] \rightarrow$ $K\left(\mathbb{E}^{i}\right), i \geq 1$, is Hukuhara differentiable at $t_{0}$, and

$$
D_{\mathrm{H}} x\left(t_{0}\right)=\lim _{\longleftarrow} D_{\mathrm{H}} x^{i}\left(t_{0}\right) .
$$

Using Theorem 3.10 and fixed point results for limit maps we will prove the following main result of this section: 
Theorem 3.13. Let $F:[0, a] \times K(\mathbb{F}) \rightarrow K(\mathbb{F})$ be a Carathéodory function, and $x_{0} \in K(\mathbb{F})$. Assume that:

(i) $F$ is a projective limit $\varliminf_{\longleftrightarrow} F^{i}$ of Carathéodory functions $F^{i}:[0, a] \times$ $K\left(\mathbb{E}^{i}\right) \rightarrow K\left(\mathbb{E}^{i}\right)$

(ii) $F$ is bounded, or equivalently, it is bounded with respect to every semimetric of $K(\mathbb{F})$, i.e., there exist $r_{i}>0$ with $D^{i}(F(t, A), 0) \leq r_{i}$ for every $i \in \mathbb{N}$,

(iii) $\left.\lim _{\delta \rightarrow 0^{+}} \alpha_{i}\left(F^{i}\left(I_{t, \delta} \times A\right)\right) \leq \omega\left(t, \alpha_{i}(A)\right)\right)$ for every bounded set $A \subset$ $K\left(\mathbb{E}^{i}\right)$ and a.e. $t \in[0, a]$, where $\alpha_{i}$ is the measure of noncompactness on $\mathbb{E}^{i}$ and $\omega$ is a Kamke function.

Then the set of solutions of $\left(C_{F}\right)$ is a nonempty $R_{\delta}$-set.

Proof. Let $x_{0}^{i}=\varrho^{i}\left(x_{0}\right)$. Consider the sequence of Cauchy problems

$$
\left(C_{F}^{i}\right)\left\{\begin{array}{l}
D_{\mathrm{H}} x^{i}(t)=F^{i}\left(t, x^{i}(t)\right) \\
x(0)=x_{0}^{i}
\end{array}\right.
$$

By the properties of the $i$-distance and assumption (ii), the mappings $F^{i}$ are bounded. Indeed,

$$
D^{i}(F(t, A), 0)=D^{i}\left(\varliminf_{\longleftarrow}^{\lim } F^{i}\left(t, A^{i}\right), \varliminf_{\longleftarrow}^{\lim } 0\right)=D^{\mathbb{E}^{i}}\left(F^{i}\left(t, A^{i}\right), 0\right)
$$

where $A=\lim _{\longleftarrow} A_{i}$.

By Theorem 3.10 the set $\mathcal{F}_{i}$ of solutions of $\left(C_{F}^{i}\right)$ is a nonempty $R_{\delta}$-set. Notice that $\mathcal{F}_{i}$, for every $i \geq 1$, is the fixed point set of the operator

$$
\Phi^{i}: C\left([0, a], K\left(\mathbb{E}^{i}\right)\right) \rightarrow C\left([0, a], K\left(\mathbb{E}^{i}\right)\right)
$$

defined by

$$
\Phi^{i}\left(x^{i}\right)(t)=x_{0}^{i}+\int_{0}^{t} F^{i}\left(s, x^{i}(s)\right) d s .
$$

Let $y \in C\left([0, a], K\left(\mathbb{E}^{i}\right)\right)$. Then

$$
\begin{aligned}
& \left(\Phi^{i} \Psi^{j i}(y)\right)(t)=x_{0}^{i}+\int_{0}^{t} F^{i}\left(s, \Psi^{j i}(y)(s)\right) d s \\
& \quad=x_{0}^{i}+\int_{0}^{t} \Psi^{j i} F^{j}(s, y(s)) d s=\Psi^{j i}\left(x_{0}^{j}+\int_{0}^{t} F^{j}(s, y(s)) d s\right)=\left(\left(\Psi^{j i} \Phi^{j}\right)(y)\right)(t)
\end{aligned}
$$

for any $j \geq i$. This means that $\left\{\Phi^{i}\right\}$ is a map of inverse systems. Set

$$
\Phi=\varliminf_{\longleftarrow} \Phi^{i}: C([0, a], K(\mathbb{F})) \rightarrow C([0, a], K(\mathbb{F})) .
$$

From Proposition 2.2 it follows that $\mathcal{F}=\operatorname{Fix} \Phi$ is a compact $R_{\delta}$. Finally, we show that $\mathcal{F}$ is indeed the set of solutions to $\left(C_{F}\right)$. Let $x \in \Phi(x)$ and write $x^{i}=\Psi^{i}(x)$ for every $i \in \mathbb{N}$. This means that $D_{\mathrm{H}} x^{i}(t)=F^{i}\left(t, x^{i}(t)\right)$ 
and $x^{i}(0)=x_{0}^{i}$ for a.e. $t \in[0, a]$ and $i \in \mathbb{N}$. By (2) we get

$$
D_{\mathrm{H}} x(t)=\lim _{\longleftarrow} D_{\mathrm{H}} x^{i}(t)=\lim _{\longleftarrow} F^{i}\left(t, x^{i}(t)\right)=F(t, x(t)) \quad \text { for a.e. } t \in[0, a]
$$

and $x(0)=x_{0}^{i}$. Conversely, it is obvious that each solution to problem $\left(C_{F}\right)$ is a fixed point of $\Phi$. The proof is complete.

\section{Topological structure of measurable-Lipschitz differential in-} clusions in Fréchet spaces. In this section we focus on the parameterized differential problem

$$
\left(C_{A F}\right)\left\{\begin{array}{l}
\dot{x}(t) \in A x(t)+F(t, x(t), \eta) \quad \text { for a.e. } t \in J=[0, a], \\
x(0)=x_{0} \in \mathbb{F}
\end{array}\right.
$$

where suitable assumptions on $A$ and $F$ will be specified below. We look for mild solutions of problem $\left(C_{A F}\right)$, i.e., for maps $x: J \rightarrow \mathbb{F}$ of the form $x(t)=T(t) x_{0}+\int_{0}^{t} T(t-s) f(s) d s$, where $f$ is a measurable selection of $F(\cdot, x(\cdot), \eta)$, and $(T(t))_{t \in J}$ is a continuous semigroup of continuous linear operators in $\mathbb{F}$. We assume that $\mathbb{F}$ is a projective limit $\lim _{\mathbb{E}}^{i}$ of Banach spaces as described in Section 2. One can check that, if each $\mathbb{E}^{i}$ is reflexive or $A=0$, then every mild solution is strong, that is, $\dot{x}(t) \in A x(t)+F(t, x(t), \eta)$ for every $\eta \in H$ and a.e. $t \in J$.

Let $\mathcal{M}_{x_{0}, \eta}$ denote the set of all mild solutions of problem $\left(C_{A F}\right)$.

TheOREM 4.1. Assume that the Banach spaces $\mathbb{E}^{i}$ are separable and the connecting morphisms $\varrho^{j i}: \mathbb{E}^{j} \rightarrow \mathbb{E}^{i}$ are surjective. We also assume

(H1) $A: \mathbb{F} \rightarrow \mathbb{F}$ is a continuous linear operator such that, for every $i \geq 1$ and $\lambda>0$,

(i) $A\left(\operatorname{Ker} p_{i}\right) \subset \operatorname{Ker} p_{i}$,

(ii) the operator $\lambda I-A$ is invertible, $(\lambda I-A)^{-1}\left(\operatorname{Ker} p_{i}\right) \subset \operatorname{Ker} p_{i}$, and $p_{i}\left((\lambda I-A)^{-1}(u)\right) \leq(1 / \lambda) p_{i}(u)$ for every $u \in \mathbb{F}$.

(H2) $F: J \times \mathbb{F} \times H \multimap \mathbb{F}$, where $H$ is a separable metric space, has closed bounded values, and is the limit map $\lim _{\longleftarrow} F^{i}$ of maps $F^{i}$ : $J \times \mathbb{E}^{i} \times H \multimap \mathbb{E}^{i}$ with closed bounded values, i.e.,

$$
\varrho^{j i}\left(F^{j}(t, x, \eta)\right)=F^{i}\left(t, \varrho^{j i}(x), \eta\right) \quad \text { for } j \geq i .
$$

Moreover, for every $i \geq 1$,

(i) $F^{i}$ is $\mathcal{L}(J) \times \mathcal{B}\left(\mathbb{E}^{i} \times H\right)$-measurable,

(ii) $F^{i}(t, x, \cdot)$ is Hausdorff continuous for every $(t, x) \in J \times \mathbb{E}^{i}$,

(iii) there are integrable functions $\alpha_{i}, \beta_{i}: J \rightarrow[0, \infty)$ such that

$$
\begin{aligned}
D^{\mathbb{E}^{i}}\left(F^{i}(t, x, \eta), F^{i}(t, y, \eta)\right) & \leq \alpha_{i}(t)\|x-y\|_{\mathbb{E}^{i}}, \\
D^{\mathbb{E}^{i}}\left(\{0\}, F^{i}(t, x, \eta)\right) & \leq \beta_{i}(t),
\end{aligned}
$$

for all $t \in J, x, y \in \mathbb{E}^{i}$ and $\eta \in H$. 
Then the set of solutions $\mathcal{M}_{x_{0}, \eta}$ is a projective limit of absolute retracts (not necessarily compact).

REMARK 4.2. The nonemptiness and $R_{\delta}$ structure of $\mathcal{M}_{x_{0}, \eta}$ can be guaranteed, as a consequence of Proposition 2.2, by convexity of values of $F^{i}$ and suitable compactness type assumptions in terms of a measure of noncompactness (see, e.g., assumption (iii) in Theorem 3.13).

Proof of Theorem 4.1. We proceed in several steps.

STEP 1. First we show that $A$ can be realized as a limit map of closed linear operators $A^{i}: \mathbb{E}^{i} \rightarrow \mathbb{E}^{i}$.

Note that the surjectivity of the connecting morphisms implies surjectivity, and also openness, of the projections $\varrho^{i}: \mathbb{F} \rightarrow \mathbb{E}^{i}$. For every $y \in \mathbb{E}^{i}=$ $\varrho^{i}(\mathbb{F})$ we choose $x \in \mathbb{F}$ such that $\varrho^{i}(x)=y$, and define $A^{i}(y):=\varrho^{i}(A x)$. To check the correctness of this definition, let $x_{1}$ and $x_{2}$ in $\mathbb{F}$ be such that $\varrho^{i}\left(x_{1}\right)=\varrho^{i}\left(x_{2}\right)$. Then

$$
A^{i} \varrho^{i}\left(x_{1}\right)-A^{i} \varrho^{i}\left(x_{2}\right)=\varrho^{i} A\left(x_{1}-x_{2}\right) \stackrel{(\mathrm{H} 1)(\mathrm{i})}{=} 0 .
$$

Now assume that $\mathbb{E}^{i} \ni u_{k} \rightarrow u$ and $A^{i} u_{k} \rightarrow v \in \mathbb{E}^{i}$. Choose any $x \in \mathbb{F}$ with $\varrho^{i}(x)=u$. For every $n \geq 1$, since $\varrho^{i}$ is open, there is a $\delta_{n}>0$ such that, if $\|y-u\|_{\mathbb{E}^{i}}<\delta_{n}$, then $y \in \varrho^{i}(\{z \in \mathbb{F}: d(z, x)<1 / n\})$. We can choose $k_{n} \geq 1$ such that $\left\|u_{k_{n}}-u\right\|_{\mathbb{E}^{i}}<\delta_{n}$, and hence there exists $x_{k_{n}} \in \mathbb{F}$ with $d\left(x_{k_{n}}, x\right)<1 / n$. From the continuity of $A$ it follows that $A x_{k_{n}} \rightarrow A x$, and consequently $A^{i} u_{k_{n}}=\varrho^{i} A x_{k_{n}} \rightarrow \varrho^{i} A x=A^{i} u$. This implies that $A^{i} u=v$, and hence $A^{i}$ is a closed operator.

Finally,

$$
\varrho^{j i} A^{j} u=\varrho^{j i} \varrho^{j} A x=\varrho^{i} A x=A^{i} \varrho^{j i} u
$$

for any $x \in \mathbb{F}$ such that $\varrho^{j}(x)=u$. Thus $\left\{A^{i}\right\}$ is a map of inverse systems.

SteP 2. We prove that $\lambda I-A^{i}$ is invertible for all $i \geq 1$ and $\lambda>0$.

For every $v \in \mathbb{E}^{i}$ we select $y \in \mathbb{F}$ with $\varrho^{i}(y)=v$. By assumption (H1)(ii) we can find $x \in \mathbb{F}$ such that $(\lambda I-A) x=y$. Set $u:=\varrho^{i}(x)$. Then

$$
v=\varrho^{i}(\lambda I-A) x=\lambda \varrho^{i}(x)-\varrho^{i} A x=\left(\lambda I-A^{i}\right) u,
$$

so the operator $\lambda I-A^{i}$ is onto.

Now, pick any $u, w \in \mathbb{E}^{i}$ and $x, z \in \mathbb{F}$ with $\varrho^{i} x=u$ and $\varrho^{i} z=w$. Assume that $\left(\lambda I-A^{i}\right) u=\left(\lambda I-A^{i}\right) w$. Then

$$
0=\left(\lambda I-A^{i}\right)(u-w)=\varrho^{i}((\lambda I-A)(x-z)),
$$

which means that $(\lambda I-A)(x-z) \in \operatorname{Ker} p_{i}$, and so $x-z \in(\lambda I-A)^{-1}\left(\operatorname{Ker} p_{i}\right)$. Assumption (H1)(ii) implies that $x-z \in \operatorname{Ker} p_{i}$. Hence, $u=w$, and the bijectivity of $\lambda I-A^{i}$ is proved. 
STEP 3. Let $R_{\lambda}^{i}=\left(\lambda I-A^{i}\right)^{-1}$, the resolvent for $A^{i}$. It is easy to check that $\left(\lambda I-A^{i}\right)^{-1}=\left((\lambda I-A)^{-1}\right)^{i}$. Taking this into account one has

$$
\begin{aligned}
\left\|R_{\lambda}^{i}(u)\right\|_{\mathbb{E}^{i}} & =\left\|\left(\lambda I-A^{i}\right)^{-1}(u)\right\|_{\mathbb{E}^{i}}=\left\|\left((\lambda I-A)^{-1}\right)^{i} u\right\|_{\mathbb{E}^{i}} \\
& =p_{i}\left((\lambda I-A)^{-1} x\right) \leq \frac{1}{\lambda} p_{i}(x)=\frac{1}{\lambda}\|u\|_{\mathbb{E}^{i}},
\end{aligned}
$$

where $\varrho^{i}(x)=u$. The Hille-Yosida theorem applied for every $i \geq 1$ implies that each $A^{i}$ is the generator of a continuous semigroup of contractions $\left(T^{i}(t)\right)_{t \in J}$. Moreover, if $j \geq i$, then

$$
\begin{aligned}
\varrho^{j i} T^{j}(t) x & =\varrho^{j i}\left(\lim _{n \rightarrow \infty}\left(I-\frac{t}{n} A^{j}\right)^{-n}(x)\right) \\
& =\lim _{n \rightarrow \infty} \varrho^{j i}\left(\left(I-\frac{t}{n} A^{j}\right)^{-n}(x)\right) \\
& =\lim _{n \rightarrow \infty}\left(I-\frac{t}{n} A^{i}\right)^{-n}(x)=T^{i}(t) x,
\end{aligned}
$$

since $\varrho^{j i}\left(I-\frac{t}{n} A^{j}\right)=\left(I-\frac{t}{n} A^{i}\right) \varrho^{j i}$, and consequently

$$
\begin{aligned}
\left(I-\frac{t}{n} A^{i}\right)^{-1} \varrho^{j i} & =\left(I-\frac{t}{n} A^{i}\right)^{-1} \varrho^{j i}\left(I-\frac{t}{n} A^{j}\right)\left(I-\frac{t}{n} A^{j}\right)^{-1} \\
& =\left(I-\frac{t}{n} A^{i}\right)^{-1}\left(I-\frac{t}{n} A^{i}\right) \varrho^{j i}\left(I-\frac{t}{n} A^{j}\right)^{-1} \\
& =\varrho^{j i}\left(I-\frac{t}{n} A^{j}\right)^{-1} .
\end{aligned}
$$

STEP 4. From Theorem 3.1 in [7] it follows that for every $i \geq 1$ the set $\mathcal{M}_{x_{0}^{i}, \eta}^{i}$ of all mild solutions of the problem

$$
\left(C_{A F}^{i}\right)\left\{\begin{array}{l}
\dot{x}(t) \in A^{i} x(t)+F^{i}(t, x(t), \eta) \quad \text { for a.e. } t \in J \\
x(0)=x_{0}^{i}=\varrho^{i}\left(x_{0}\right) \in \mathbb{F}
\end{array}\right.
$$

is an absolute retract, being a retract of the convex closed subset

$$
\left\{x: J \rightarrow \mathbb{E}^{i} \mid x(t)=T^{i}(t) x_{0}+\int_{0}^{t} T^{i}(t-s) f(s) d s, f \in L^{1}\left(J, \mathbb{E}^{i}\right)\right\}
$$

of the Banach space

$W^{i}\left(J, \mathbb{E}^{i}\right)$

$$
:=\left\{x: J \rightarrow \mathbb{E}^{i} \mid x(t)=T^{i}(t) \xi+\int_{0}^{t} T^{i}(t-s) f(s) d s, \xi \in \mathbb{E}^{i}, f \in L^{1}\left(J, \mathbb{E}^{i}\right)\right\}
$$

equipped with the norm $\|x\|_{W^{i}}=\|x\|_{C}+\|f\|_{L^{1}}$. Here $\|f\|_{L^{1}}$ is the weighted Bielecki norm (cf. [7]). 
Let $x_{0}=\left(x_{0}^{i}\right) \in \mathbb{F}$, where $x_{0}^{i} \in \mathbb{E}^{i}$. We check that $\left\{\mathcal{M}_{x_{0}, \eta}^{i}\right\}$ is an inverse system. Let $x \in \mathcal{M}_{x_{0}^{j}, \eta}^{j}$ and $i \leq j$. Then, since the connecting morphisms are linear,

$$
\begin{aligned}
\varrho^{j i} x(t) & =\varrho^{j i}\left(T^{j}(t) x_{0}^{j}\right)+\varrho^{j i}\left(\int_{0}^{t} T^{j}(t-s) f(s) d s\right) \\
& =T^{i}(t) \varrho^{j i}\left(x_{0}^{j}\right)+\int_{0}^{t} T^{i}(t-s) \varrho^{j i} f(s) d s
\end{aligned}
$$

for some $f(\cdot) \in F^{j}(\cdot, x(\cdot), \eta)$. But

$$
\varrho^{j i} f(s) \in \varrho^{j i}\left(F^{j}(s, x(s), \eta)\right)=F^{i}\left(s, \varrho^{j i} x(s), \eta\right),
$$

so $\varrho^{j i} f$ is a measurable selection, and this implies that $\varrho^{j i} \in \mathcal{M}_{x_{0}, \eta}^{i}$. By Step 3 there exists a family of limit operators $T(t)=\lim ^{i} T^{i}(t)$. Assume that $f$ is a measurable selection of $F(\cdot, x(\cdot), \eta)$, and

$$
x(t)=T(t) x_{0}+\int_{0}^{t} T(t-s) f(s) d s,
$$

i.e., $x \in \mathcal{M}_{x_{0}, \eta}$. Then we can check as above that $\varrho^{i} x \in \mathcal{M}_{\varrho^{i}\left(x_{0}\right), \eta}^{i}$ for every $i \geq 1$. On the other hand, if $\left(f^{i}\right)_{i \geq 1}$ is a thread of measurable maps $f^{i}: I \rightarrow \mathbb{E}^{i}$ such that $f^{i}(s) \in F^{i}\left(s, x^{i}(s), \eta\right)$ for a.e. $s \in I$, where $\left(x^{i}\right)_{i \geq 1}$ is a thread in $\left\{\mathcal{M}_{x_{0}^{i}, \eta}^{i}\right\}$, then $f:=\lim _{\longleftarrow} f^{i}: I \rightarrow \mathbb{F}$ is a measurable selection of $F\left(\cdot, \lim ^{i} x^{i}(\cdot), \eta\right)$. Indeed, for every base open subset $\left(\varrho^{i}\right)^{-1}(U)$ of $\mathbb{F}$, where $U$ is open in $\mathbb{E}^{i}$, the set $f^{-1}\left(\left(\varrho^{i}\right)^{-1}(U)\right)=\left(\varrho^{i} f\right)^{-1}(U)=\left(f^{i}\right)^{-1}(U)$ is measurable. Moreover, since $f^{i} \in F^{i}\left(\cdot, x^{i}(\cdot), \eta\right)$, we obtain

$$
f=\lim f^{i} \in \lim _{\longleftarrow} F^{i}\left(\cdot, x^{i}(\cdot), \eta\right)=F\left(\cdot, \lim x^{i}(\cdot), \eta\right) .
$$

Now, for $x:=\lim _{\longleftarrow} x^{i}$, we have $\varrho^{i}(x(t))=T^{i}(t) x_{0}^{i}+\int_{0}^{t} T^{i}(t-s) f^{i}(s) d s$, and consequently $\overleftarrow{x(t)}=T(t) x_{0}+\int_{0}^{t} T(t-s) f(s) d s$, that is, $x \in \mathcal{M}_{x_{0}, \eta}$. Thus $\mathcal{M}_{x_{0}, \eta}=\lim _{\longleftarrow} \mathcal{M}_{x_{0}^{i}, \eta}^{i}$. This completes the proof of the theorem.

REMARK 4.3. In the above result we can assume, instead of (H1), that $A=\lim A^{i}$ where each $A^{i}: D\left(A^{i}\right) \rightarrow \mathbb{E}^{i}$ is the generator of a continuous semigroup of bounded linear operators. Notice that Steps 1-3 of the proof were devoted to showing this projective limit connection between $A$ and $A^{i}$. Note that since $\overline{D\left(A^{i}\right)}=\mathbb{E}^{i}$ and $\lim _{\longleftarrow} D\left(A^{i}\right)=D(A)$, the operator $A$ is also densely defined and closed.

Open problems. 1. Is the solution set $\mathcal{M}_{x_{0}, \eta}$ an absolute retract? This is an open problem even in the case where the values of $F$ are convex and 
compact. It seems that a positive answer depends on the possibility of constructing a map $\left\{r^{i}: L^{1}\left(I, \mathbb{E}^{i}\right) \rightarrow \mathcal{M}_{x_{0}^{i}, \eta}^{i}\right\}$ of inverse systems consisting of retractions. The existence of such retractions on each level follows from results based on the method due to Bressan, Cellina and Fryszkowski [4]. The difficulty is to guarantee that $\varrho^{j i} r^{j}=r^{i} \varrho^{j i}$. We know that in general the choice of a sequence of retractions connected by such equalities is impossible, and hence usually projective limits of absolute retracts are only $R_{\delta}$ sets.

2. Does there exist a continuous selection $\eta$ of the solution map $x_{0} \mapsto$ $\mathcal{M}_{x_{0}, \eta}$ ? The obstacles to solving this problem are similar. Note that the answer is affirmative in the case of a Banach space (for a right-hand side $F(t, x) \equiv F(x))$ even if we only assume that $F$ is one-sided Lipschitz continuous (see [11]).

\section{References}

[1] J. Andres, G. Gabor and L. Górniewicz, Topological structure of solution sets to multivalued asymptotic problems, Z. Anal. Anwend. 19 (2000), 35-60.

[2] A. Bąkowska, Topological approach to ordinary differential equations and fixed point results in a class of metric spaces, to appear.

[3] H. T. Banks and M. Q. Jacobs, A differential calculus for multifunctions, J. Math. Anal. Appl. 29 (1970), 246-272.

[4] A. Bressan, A. Cellina and A. Fryszkowski, A class of absolute retracts in spaces of integrable functions, Proc. Amer. Math. Soc. 111 (1991), 413-418.

[5] T. F. Bridgland, Trajectory integrals of set valued functions, Pacific J. Math. 33 (1970), 43-68.

[6] F. Browder and C. P. Gupta, Topological degree and nonlinear mappings of analytic type in Banach spaces, J. Math. Anal. Appl. 26 (1969), 390-402.

[7] F. De Blasi, G. Pianigiani and V. Staicu, Topological properties of nonconvex differential inclusions of evolution type, Nonlinear Anal. 24 (1995), 711-722.

[8] K. Deimling, Ordinary Differential Equations in Banach Spaces, Lecture Notes in Math. 596, Berlin, Springer, 1977.

[9] R. Engelking, Outline of General Topology, North-Holland and PWN, 1968.

[10] G. Gabor, On the acyclicity of fixed point sets of multivalued maps, Topol. Methods Nonlinear Anal. 14 (2000), 327-343.

[11] - Continuous selection of the solution map to one-sided Lipschitz differential inclusions, Nonlinear Anal. 66 (2007), 1185-1197.

[12] G. N. Galanis, T. Gnana Bhaskar and V. Lakshmikantham, Set differential equations in Fréchet spaces, J. Appl. Anal. 14 (2008), 103-113.

[13] G. N. Galanis, T. Gnana Bhaskar, V. Lakshmikantham and P. K. Palamides, Set valued functions in Fréchet spaces: Continuity, Hukuhara differentiability and applications to set differential equations, Nonlinear Anal. 61 (2005), 559-575.

[14] L. Hörmander, Sur la fonction d'appui des ensembles convexes dans un espace localement convexe, Ark. Mat. 3 (1955), 181-186.

[15] M. Kisielewicz, Multivalued differential equations in separable Banach spaces, J. Optim. Theory Appl. 37 (1982), 231-249. 
[16] D. O'Regan, An essential map approach for multimaps defined on closed subsets of Fréchet spaces, Appl. Anal. 85 (2006), 503-513.

[17] H. Rådström, An embedding theorem for spaces of convex sets, Proc. Amer. Math. Soc. 3 (1952), 165-169.

[18] A. Tolstonogov, Differential Inclusions in a Banach Space, Math. Appl. 524, Kluwer, Dordrecht, 2000.

Faculty of Mathematics and Computer Science

Nicolaus Copernicus University

Chopina $12 / 18$

87-100 Torun, Poland

E-mail: abak@neostrada.pl

ggabor@mat.uni.torun.pl

Received 11.2.2008

and in final form 14.7.2008 